\title{
Lorsque l'industrie dévoile ses relations avec le corps médical
}

\section{Hermann Amstad a,}

Walter H. Reinhart ${ }^{b}$

a Dr, Secrétaire général de l'ASSM, Bâle

b Prof. Dr, ancien médecin chef du service de médecine interne, Hôpital cantonal des Grisons, Coire, et Président de la «Commission consultative»
Correspondance:

ASSM

Petersplatz 13

CH-4051 Bâle

mail[at]samw.ch
Voici bientôt 15 ans que l'Académie Suisse des Sciences Médicales (ASSM) a amorcé le débat autour de la collaboration entre le corps médical et l'industrie. Des experts suisses et étrangers étaient venus exposer leur point de vue sur ce thème sensible dans le cadre d'un workshop, au siège de l'Académie à Bâle. Par la suite, le comité de direction de l'ASSM avait chargé deux groupes de travail d'élaborer des recommandations concernant les domaines de la «Recherche clinique» et de la «Formation postgraduée et continue». Au moment de leur parution, le corps médical a pris connaissance de ces recommandations avec un désintérêt courtois.

Mais, lorsque, trois ans plus tard, l'ASSM décidait de réviser ces recommandations et - plus important encore - de les publier en tant que directives, la situation a connu un brusque revirement. Lors de la consultation, nombreuses étaient les réactions virulentes. La majorité des critiques concernaient le chapitre «Formation postgraduée et continue», notamment l'interdiction du monosponsoring des sessions de formation continue et l'obligation de s'acquitter d'un droit d'inscription pour chaque session de formation. Ces directives ont été intégrées dans le Code de déontologie de la FMH en 2006 et revêtent, de ce fait, un caractère contraignant pour le corps médical.

\section{Missions accomplies pour la Commission}

En 2006, l'ASSM a instauré une «commission consultative» pour l'application des directives «Collaboration corps médical-industrie». Depuis lors, cette commission soutient la mise en pratique et l'interprétation des directives. Très nombreuses au début, les demandes ont peu à peu diminué par la suite.

Une demande d'intérêt général, parvenue au cours de l'année passée, est brièvement relatée ciaprès: L'ASSM a-t-elle émis des consignes pour le sponsoring d'une société de discipline ou d'une fondation propre à la société de discipline? Si les directives de l'ASSM, actuellement en vigueur, ne règlent pas explicitement ce point, elles donnent néanmoins quelques consignes pouvant être appliquées à cette situation:

- Les contrats de sponsoring sont conclus par écrit; ils règlent les obligations mutuelles.

- Il y a plusieurs sponsors indépendants les uns des autres.

- Les fonds provenant de sponsors sont comptabilisés sur un compte institutionnel prévu à cet effet.

- Les sommes perçues ou versées sont révélées de façon transparente.
La pratique a régulièrement révélé un certain nombre de lacunes et d'imprécisions dans les directives, c'est pourquoi, l'ASSM a décidé de les réviser en 2012. Ce faisant, différents paragraphes ont été remaniés et complétés et le chapitre «Activité d'experts» a été ajouté. Avec la révision des directives, la Commission consultative considère qu'elle a rempli son mandat et a, de ce fait, sollicité sa dissolution. Lors de sa séance de fin novembre, le Sénat de l'ASSM a accepté cette demande et dissous la commission au 31 décembre 2013 en remerciant ses membres pour les services rendus; le «poste de consultation» au secrétariat général de l'ASSM continuera toutefois de répondre aux éventuelles demandes.

\section{La signification du nouveau «Code de coopération pharmaceutique»}

La dissolution de la commission a été acceptée plus facilement par le Sénat grâce à l'entrée en vigueur du nouveau «Code de coopération pharmaceutique» qui apporte un soutien bienvenu aux directives. En 2013, les Etats-Unis ont adopté le «Physician Payment Sunshine Act», par lequel les entreprises s'engagent à consigner tous leurs paiements aux fournisseurs de prestations médicales dans un registre accessible au public. Des dispositions similaires existent déjà en France et dans d'autres pays européens et l'UE a signalé qu'elle souhaitait également promulguer de telles réglementations. Dans ce contexte, l'European Federation of Pharmaceutical Industries and Associations (EFPIA) a décidé d'agir et d'adopter ses propres réglementations (suite à quoi l'UE a renoncé à édicter ses propres règles). Les membres de l'EFPIA ont pour tâche d'adapter les prescriptions européennes aux conditions nationales; c'est pourquoi scienceindustries, Interpharma et vips (association des entreprises pharmaceutiques en Suisse) ont adopté récemment le Code de coopération pharmaceutique qui prévoit de publier à partir de 2015, également en Suisse, tous les paiements aux fournisseurs de prestations médicales. Dès lors, le leitmotiv des directives de l'ACP de 1990, dont s'étaient inspirées les recommandations et les directives, devient soudain réalité: «A useful criterion in determining acceptable activities and relationships is: would you be willing to have these arrangements generally known?» Ainsi, dans la «collaboration corps médical - industrie», l'industrie a repris le flambeau pour franchir un pas de plus, une occasion pour le corps médical de s'y adapter et, éventuellement, de repenser certains modes de comportement. 